CLINICAL PRACTICE Clinical Vignettes

\title{
A Case of Pseudothrombotic Microangiopathy Associated with Pernicious Anemia
}

\author{
Henri Wathieu, BA and Kristin M. Bateman, MD D \\ John W. Deming Department of Medicine, Tulane University School of Medicine, New Orleans, LA, USA.
}

J Gen Intern Med 36(6):1775-7

DOI: $10.1007 / \mathrm{s} 11606-020-06588-2$

(C) Society of General Internal Medicine 2021

\section{INTRODUCTION}

Pernicious anemia (PA) is the result of cobalamin (vitamin $\mathrm{B}_{12}$ ) deficiency in the setting of chronic autoimmune gastritis. The underlying pathophysiology of PA involves antibody formation against intrinsic factor (IF) or the gastric parietal cells which produce it, preventing adequate absorption of vitamin $\mathrm{B}_{12}$ at the terminal ileum. ${ }^{1}$ This deficiency halts a number of metabolic processes, including DNA synthesis and myelinogenesis. ${ }^{1}$ After a latency period of vitamin $\mathrm{B}_{12}$ storage depletion, patients with PA develop typical symptoms of anemia such as fatigue, pallor, tachycardia, and lightheadedness. ${ }^{2}$ Progressive neuro-psychiatric symptoms occur, including peripheral numbness and paresthesias with eventual weakness, ataxia, and mental disturbances. ${ }^{2}$ Diagnostic criteria vary, but typically include laboratory evidence of anemia, low levels of vitamin $\mathrm{B}_{12}$, macrocytosis with hypersegmented neutrophils in peripheral blood, histological evidence of gastric atrophic lesions, and the presence of antiIF or anti-parietal cell antibodies. ${ }^{2}$

Hemolysis is a rare hematologic manifestation in PA, occurring in approximately $1.5 \%$ of cases. ${ }^{3}$ The impaired hematopoiesis of vitamin $\mathrm{B}_{12}$ deficiency can itself result in a hemolytic picture, and this must be recognized as distinct from other rare etiologies of hemolysis in PA, including pseudothrombotic microangiopathy (pseudo-TMA) and autoimmune hemolytic anemia (AIHA) ${ }^{3,4}$ Here, we present a case of pseudo-TMA, a microangiopathic phenomenon in PA marked by the triad of hemolytic anemia, thrombocytopenia, and schistocytosis in the setting of vitamin $\mathrm{B}_{12}$ deficiency. This clinical vignette highlights the ways in which pseudoTMA can be distinguished from primary causes of thrombotic microangiopathy (whose features it mimics) and from other causes of hemolysis in PA.

Received August 24, 2020

Accepted December 30, 2020

Published online February 23, 2021

\section{CASE PRESENTATION}

A 53-year-old woman with a history of osteoarthritis and anemia presented with shortness of breath at rest for 2 weeks that was worse on exertion. She reported severe fatigue and malaise for the past 3 months, along with intermittent episodes of lightheadedness and headaches without known triggers. She also complained of longstanding bilateral leg pain and foot numbness. Her home medications were tramadol for low back pain and gabapentin for leg pain. She recalled being first diagnosed with anemia in the 1990s, when she received a single blood transfusion and began receiving subcutaneous vitamin $\mathrm{B}_{12}$ injections daily, then weekly, then monthly, with her last dose being approximately 1 year prior to presenting.

The patient was afebrile with a blood pressure of 124/57 $\mathrm{mm} \mathrm{Hg}$, pulse of 83 beats per minute, and a respiratory rate of $18 /$ minute with oxygen saturation of $97 \%$ on room air. Physical examination was notable for conjunctival pallor and $1+$ pitting edema of the lower extremities bilaterally to the level of the lower shin. There were no associated petechiae or purpura of the skin or oral mucosa. Cardiac auscultation revealed a holosystolic flow murmur. Laboratory testing (Table 1) showed mild leukopenia, severe anemia, thrombocytopenia, and erythrocyte indices suggestive of macrocytosis. The severe symptomatic anemia with a hemoglobin level of $5 \mathrm{~g} / \mathrm{dL}$ prompted transfusion of 2 units of packed red blood cells. A chest $\mathrm{x}$-ray was unremarkable. Renal and liver function tests, including total bilirubin and coagulation studies, were normal.

Additional studies (Table 1) were aimed at narrowing the differential diagnosis of our patient's severe anemia and pancytopenia. A peripheral blood smear (PBS) showed 1+ polychromasia and anisocytosis as well as $2+$ macrocytosis, schistocytosis, poikilocytosis, and spherocytosis. A low corrected reticulocyte count of $0.5 \%$ suggested a hypoproliferative etiology. Iron studies revealed an elevated ferritin which ruled out iron deficiency or occult bleed, while a high lactate dehydrogenase (LDH) and low haptoglobin were suggestive of an active hemolytic process. Notably, vitamin $\mathrm{B}_{12}$ levels were below the detection limit, with normal folate. Serum anti-intrinsic factor and anti-parietal cell antibodies were present. The thyroid-stimulating hormone level was nor$\mathrm{mal}$ at $1.11 \mathrm{mIU} / \mathrm{mL}$. Infectious work-up was performed, wherein serology suggested previous EBV infection and a negative hepatitis panel. CMV, influenza, and HIV tests were nonreactive. Serum and urine protein electrophoresis were 
Table 1 Select Laboratory Findings

\begin{tabular}{lll}
\hline \hline Test & Result & Reference range \\
\hline Leukocyte count $(/ \mathrm{mL})$ & 3600 & $4500-11,000$ \\
Hemoglobin $(\mathrm{g} / \mathrm{dL})$ & 5.0 & $12.0-16.0$ \\
Hematocrit $(\%)$ & 15.1 & $36.0-46.0$ \\
Platelet count $(/ \mu \mathrm{L})$ & 106,000 & $160,000-420,000$ \\
Mean corpuscular volume $(\mathrm{fL})$ & 116.6 & $80.0-100.0$ \\
Red blood cell distribution width $(\%)$ & 27.6 & $12.0-15.0$ \\
Corrected reticulocyte count $(\%)$ & 0.5 & $1.1-3.3$ \\
Serum iron $(\mu \mathrm{g} / \mathrm{dL})$ & 151 & $50-170$ \\
Total iron binding capacity $(\mu \mathrm{g} / \mathrm{dL})$ & 244 & $250-450$ \\
Ferritin $(\mathrm{ng} / \mathrm{mL})$ & 408.4 & $8-252$ \\
Erythrocyte sedimentation rate $(\mathrm{mm} / \mathrm{h})$ & 74 & $0-30$ \\
C-reactive protein $(\mathrm{mg} / \mathrm{dL})$ & $<0.29$ & $<3.0$ \\
Lactate dehydrogenase $(\mathrm{U} / \mathrm{L})$ & 4418 & $84-246$ \\
Haptoglobin $(\mathrm{mg} / \mathrm{dL})$ & $<8.0$ & $30-200$ \\
Vitamin $\mathrm{B}_{12}(\mathrm{pg} / \mathrm{mL})$ & $<60$ & $193-986$ \\
Folate $(\mathrm{ng} / \mathrm{mL})$ & 17.5 & $3.0-17.5$ \\
\hline
\end{tabular}

within normal limits. Other autoimmune studies including anti-nuclear antibodies and direct antiglobulin test were negative.

Following a blood transfusion, she was started on high-dose oral vitamin $\mathrm{B}_{12}(2000 \mu \mathrm{g})$ daily. Her home dose of gabapentin was also initiated for neuropathic lower extremity pain. This treatment resulted in symptomatic improvement in her fatigue and pain, and her hemoglobin level improved to $7.9 \mathrm{~g} / \mathrm{dL}$ before she was discharged. She was referred for an outpatient esophagogastroduodenoscopy.

\section{DISCUSSION}

This patient had a clinical presentation, medical history, and initial labs highly suggestive of vitamin $\mathrm{B}_{12}$ deficiency. Nonetheless, the presence of hemolytic anemia and pancytopenia were concerning and warranted a relatively broad work-up. The severely low vitamin $\mathrm{B}_{12}$ level and PBS consistent with megaloblastic anemia confirmed our initial suspicion, and a diagnosis of PA was made following tests showing anti-IF and anti-parietal cell antibodies. Concurrent pancytopenia is found in approximately $5 \%$ of cases of PA. ${ }^{3}$ Patients with hemolytic anemia in PA usually have some degree of indirect hyperbilirubinemia, ${ }^{5}$ which was not present in this case. But because erythrocyte precursors produce little bilirubin, normal levels of total bilirubin with a markedly elevated LDH and low haptoglobin are consistent with intramedullary hemolysis secondary to vitamin $\mathrm{B}_{12}$ deficiency. ${ }^{6,7} \mathrm{AIHA}$, which was ruled out in our patient with a negative direct Coombs test, has been shown to co-exist with PA in rare cases. ${ }^{8-11}$ Her improvement with continued vitamin $B_{12}$ supplementation further suggested that concurrent AIHA was unlikely, as true AIHA typically requires glucocorticoids, splenectomy, or other immunosuppressive agents for adequate response. ${ }^{12}$

Pseudo-TMA is a rare manifestation of PA that occurs in approximately $2.5 \%$ of cases of vitamin $\mathrm{B}_{12}$ deficiency. ${ }^{3}$ Its prevalence is higher in PA compared to nutritive causes, and this is likely because PA represents a more severe deficiency, with lower average serum levels of both vitamin $\mathrm{B}_{12}$ and hemoglobin. ${ }^{3}$ The pathogenesis of pseudo-TMA is not well understood, but it may be linked to the buildup of homocysteine that characterizes vitamin $\mathrm{B}_{12}$ deficiency. ${ }^{13,14}$ Homocysteine has previously been shown to directly damage the vascular endothelium by an oxidative process. ${ }^{15}$ Endothelial dysfunction, in turn, results in activation of the coagulation cascade and associated platelet aggregation. ${ }^{15}$ This suggests a true thrombotic microangiopathic mechanism for fragmentation of erythrocytes in pseudo-TMA.

The typical features of pseudo-TMA mimic those of thrombotic thrombocytopenic purpura (TTP), including thrombocytopenia, hemolytic anemia, and schistocytosis. ${ }^{16}$ TTP is a familial or autoimmune disorder in which von Willebrand factor multimers cannot be cleaved, causing platelet microthrombi to form in the microvasculature. ${ }^{17}$ It is imperative to differentiate pseudo-TMA from TTP, as TTP is immediately life-threatening and requires treatment with therapeutic plasma exchange (TPE). There are a number of laboratory parameters that aid in distinguishing the two. Notably, patients with TTP will exhibit a bone marrow response to microangiopathic hemolysis evidenced by reticulocytosis. Because vitamin $\mathrm{B}_{12}$ deficiency disrupts hematopoiesis, an adequate reticulocyte response cannot be mounted and the corrected reticulocyte count will remain low, as in this patient. ${ }^{16}$ Additionally, patients with pseudoTMA have been shown to have higher median platelet counts and LDH levels compared to those with TTP. ${ }^{6,16,18}$ The PLASMIC score is an algorithm that allows investigators to accurately estimate the pre-test probability of TTP from rapidly accessible labs and clinical factors, considering seven variables in patients with evidence of thrombotic microangiopathy. ${ }^{19,20}$ In brief, these variables include a low platelet count, evidence of hemolysis, the absence of active cancer, the absence of a stem cell transplant or solid organ transplant, a low MCV, a low international normalized ratio (INR), and a low creatinine level. $\mathrm{An} \mathrm{MCV}<90 \mathrm{fL}$ and a platelet count $<30,000 / \mu \mathrm{L}$ are two notable parameters associated with TTP that our patient did not meet, suggesting that these are important factors in the differential diagnosis of thrombotic microangiopathy. Our patient's PLASMIC score was 3, positioning her as low risk for severe ADAMTS13 deficiency.

In reviewing the literature, we were unable to find documented cases of true TTP co-occurring with vitamin $\mathrm{B}_{12}$ deficiency. Despite the low prevalence of pseudo-TMA, it is imperative that vitamin $\mathrm{B}_{12}$ deficiency be considered in the face of TTP diagnosed with one or more equivocal labs. This is true particularly if the reticulocyte response is inadequate in the setting of microangiopathic hemolytic anemia, as this may suggest pseudo-TMA and result in unnecessary treatment with TPE, which can itself herald major complications. ${ }^{21,22}$ Pseudo-TMA typically responds to vitamin $\mathrm{B}_{12}$ replenishment, and our patient responded well to $2000 \mu \mathrm{g}$ daily of oral vitamin $\mathrm{B}_{12}$, which has been shown to be equivalent in efficacy to periodic intramuscular or subcutaneous injections by way of passive diffusion in the small intestine independent of intrinsic factor. ${ }^{23}$ 
Clinicians should recognize that in severe cases of PA, a spectrum of hematological abnormalities may manifest in approximately $10 \%$ of cases. ${ }^{3}$ These include hemolytic anemia, pancytopenia, pseudo-TMA, or a combination of these. Evidence of hemolysis should prompt testing for antierythrocyte antibodies by Coombs testing to rule out AIHA, which rarely co-occurs with PA. Pseudo-TMA is a rare but under-recognized cause of thrombotic microangiopathy in vitamin $\mathrm{B}_{12}$ deficiency presenting with a triad of hemolytic anemia, schistocytosis, and thrombocytopenia, which can be mistaken for a primary thrombotic microangiopathy such as TTP. When these clinical features arise, the reticulocyte response is particularly helpful in distinguishing between hematologic sequelae that arise from impaired hematopoiesis (as in PA and associated pseudo-TMA) compared to TTP.

Acknowledgments: The authors would like to acknowledge Marjorie Bateman, M.D., and Ian Gold, M.D., for their involvement in the care of this patient.

Corresponding Author: Kristin M. Bateman, MD; John W. Deming Department of Medicine, Tulane University School of Medicine, New Orleans, LA, USA (e-mail: kbateman@tulane.edu).

\section{Compliance with Ethical Standards:}

Conflict of Interest: The authors declare that they do not have a conflict of interest.

\section{REFERENCES}

1. Toh BH. Chapter 44, Autoimmune gastritis and pernicious anemia. In: The Autoimmune Diseases. Amsterdam: Academic Press; 2020:833-47.

2. Bizzaro N, Antico A. Diagnosis and classification of pernicious anemia. Autoimmun Rev. 2014;13:565-68.

3. Andrès $\mathbf{E}$, Affenberger $\mathbf{S}$, Zimmer $\mathbf{J}$, et al. Current hematological findings in cobalamin deficiency. A study of 201 consecutive patients with documented cobalamin deficiency. Clin Lab Haematol. 2006;28:50-6.

4. Pirofsky B, Vaughn M. Addisonian pernicious anemia with positive antiglobulin tests: A multiple autoimmune disease syndrome. Am J Clin Pathol. 1968;50:459-66.

5. Yokoyama K, Ikeda Y. Autoimmune Hematological Diseases. Japan Med Assoc J. 2004;47:412-8.

6. Buess CS, Germann AM, Maloney EP, Mohammed AB. Vitamin B12 Deficiency with Pseudothrombotic Microangiopathy and Thrombotic Thrombocytopenic Purpura: Similarities and Differences. Kans J Med, 2020;13:46.
7. Garderet L, Maury E, Lagrange M, Najman A, Offenstadt G, Guidet B. Schizocytosis in pernicious anemia mimicking thrombotic thrombocytopenic purpura. Am J Med. 2003;114:423-5.

8. Yeruva SLH, Manchandani RP, Oneal P. Pernicious anemia with autoimmune hemolytic anemia: a case report and literature review. Case Rep Hematol. 2016;2016:7231503.

9. Zafad S, Madani A, Harif M, Quessar A, Benchekroun S. Pernicious anemia associated with autoimmune hemolytic anemia and alopecia areata. Pediatr Blood Cancer, 2007;49:1017-8.

10. Baba AA, Maharaj D. Hypocalcaemia in autoimmune haemolytic anaemia and pernicious anaemia. Postgrad Med J. 1988;64:61-2.

11. Salvidio E, Venzano C, Boccaccio P, Ravazzolo R, Gaetani GF, Ajmar F. Pernicious anaemia followed by autoimmune haemolytic anaemia. Proc R Soc Med. 1975;68:421.

12. Rabinowitz AP, Sacks Y, Carmel R. Autoimmune cytopenias in pernicious anemia: a report of four cases and review of the literature. Eur J Haematol. 1990;44:18-23.

13. Acharya U, Gau JT, Horvath W, Ventura P, Hsueh CT, Carlsen W. Hemolysis and hyperhomocysteinemia caused by cobalamin deficiency: three case reports and review of the literature. J Hematol Oncol. 2008;1:26.

14. Chapuis TM, Favrat B, Bodenmann P. Cobalamin deficiency resulting in a rare haematological disorder: a case report. J Med Case Rep. 2009;3:80.

15. Nappo F, De Rosa N, Marfella R, et al. Impairment of endothelia functions by acute hyperhomocysteinemia and reversal by antioxidant vitamins. JAMA. 1999;281:2113-8.

16. Noël N, Maigné G, Tertian G, et al. Hemolysis and schistocytosis in the emergency department: consider pseudothrombotic microangiopathy related to vitamin B12 deficiency. QJM. 2013;106:1017-22.

17. George JN. Thrombotic thrombocytopenic purpura. N Engl J Med. 2006;354: 1927-35.

18. Walter $\mathbf{K}$, Vaughn $\mathbf{J}$, Martin $\mathbf{D}$. Therapeutic dilemma in the management of a patient with the clinical picture of TTP and severe B 12 deficiency. BMC Hematol. 2015;15: 16.

19. Bendapudi PK, Hurwitz S, Fry A, et al. Derivation and external validation of the PLASMIC score for rapid assessment of adults with thrombotic microangiopathies: a cohort study. Lancet Haematol. 2017;4:e157-e164

20. Li A, Khalighi PR, Wu Q, Garcia DA. External validation of the PLASMIC score: a clinical prediction tool for thrombotic thrombocytopenic purpura diagnosis and treatment. J Thromb Haemost. 2018;16:164-9.

21. Yousaf F, Spinowitz B, Charytan C, Galler M. Pernicious anemia associated cobalamin deficiency and thrombotic microangiopathy: case report and review of the literature Case Rep Med. 2017;2017:9410727..

22. Som S, Deford CC, Kaiser ML, et al. Decreasing frequency of plasma exchange complications in patients treated for thrombotic thrombocytopenic purpura-hemolytic uremic syndrome, 1996 to 2011. Transfusion. 2012;52:2525-32.

23. Carmel R. Current concepts in cobalamin deficiency. Annu Rev Med. 2000;51:357-75.

Publisher's Note Springer Nature remains neutral with regard to jurisdictional claims in published maps and institutional affiliations. 